\title{
OPTIMIERUNG VON REGELMENGEN IN EXPERTENSYSTEMEN DURCH ANALYSE ÄHNLICHER UND PLAUSIBLER FÄLLE
}

\author{
Heribert Schimpe, RWTH Aachen
}

Viele Expertensystem-Projekte werden nach der Erstellung eines Prototypen abgebrochen. Oftmals liegt der Grund hierfür in der Schwierigkeit, auch die "komplizierteren" Fälle und Ausnahmesituationen zu benennen und in die Regelmenge zu integrieren. Aufgrund der Komplexität dieses Problems besteht zunehmend Bedarf nach vollautomatischen Verfahren zur Optimierung einer Regelmenge in Expertensystemen.

Dabei ist insbesondere die Verwendung von "Fällen", d.h. von Beispielsituationen, bekannt. Hierzu existieren bereits Verfahren aus dem Bereich des maschinellen Lernens, die entweder einzelne Fälle analysieren und das Ergebnis zu verallgemeinern versuchen oder eine Vielzahl von Fällen auf Gemeinsamkeiten untersuchen. Ergebnis ist in jedem Fall die Generierung neuer Regeln.

Im vorliegenden Beitrag wird aufgezeigt, daß zum einen "ähnliche" Fälle bei der Entscheidungsfindung eines Expertensystems hilfreich sein können und zum anderen eine "plausible Ähnlichkeit" definiert werden kann, die insbesondere bei der Suche nach falschem oder unvollständigem Wissen von Nutzen ist.

Wie auch bei den in der Literatur weit verbreiteten Verfahren des Erklärungsbasierten Lernens geschieht die Analyse eines Falles über den Aufbau eines sogenannten Erklärungsbaumes. Zur Nutzung von als "ähnlich" klassifizierten Fällen in neuen Anwendungssituationen ist eine spezielle Darstellung des Erklärungsbaumes notwendig, um auf alle Elemente des Baumes gezielt zugreifen und eine vorwärtsgerichtete Inferenz simulieren zu können.

Ähnlichkeit von Fällen wird hier in einer deduktiven Form definiert, d.h. als "beweisbare" Ähnlichkeit, die anders als induktive Ähnlichkeit auf Vermutungen verzichten kann. Dabei liegt die Philosophie zugrunde, daß zwei Fälle nur dann ähnlich sein können, wenn sie wenigstens in Teilaspekten identisch sind.

Plausible Erklärungen eines Falles sind nicht deduktiv herleitbar, sondern haben einen induktiven Charakter: es handelt sich lediglich um eine Hypothese, daß die Erklärung Gültigkeit besitzt; im allgemeinen allerdings eine Vermutung mit hoher Wahrscheinlichkeit. Zum Aufbau einer plausiblen Erklärung wird die Verwendung (induktiver) Default-Regeln vorgeschlagen. Plausible Erklärungen sind einerseits eine "Rettung" in Situationen, in denen das Hintergrundwissen nicht ausreicht, eine exakte (deduktive) Erklärung zu finden, vor allem aber sind plausible Erklärungen Indizien und Ansatzpunkte für falsches oder unvollständiges Wissen in Expertensystemen und können somit eine tiefliegende Wissensrevidierung oder -erweiterung leiten. 